\title{
HOMOTOPY GROUPS OF THE SPACE OF CURVES ON A SURFACE
}

\author{
VLADIMIR TCHERNOV
}

\begin{abstract}
We explicitly calculate the fundamental group of the space $\mathscr{F}$ of all immersed closed curves on a surface $F$. It is shown that $\pi_{n}(\mathscr{F})=0, n \geq 2$, for $F \neq S^{2}, \mathrm{R} P^{2}$. It is also proved that $\pi_{2}(\mathscr{F})=\mathrm{Z}$, and $\pi_{n}(\mathscr{F})=\pi_{n}\left(S^{2}\right) \oplus \pi_{n+1}\left(S^{2}\right), n \geq 3$, for $F$ equal to $S^{2}$ or $\mathrm{R} P^{2}$.
\end{abstract}

By a surface we mean any smooth two-dimensional manifold.

\section{Introduction}

Recently the space of closed curves on a surface attracted a lot of attention. The interest was initiated by the work of V. Arnold [1], who axiomatically defined invariants St and $J^{ \pm}$of generic curves on $\mathrm{R}^{2}$.

In order to define axiomatically this kind of invariants on an arbitrary surface $F$ one has to know the fundamental group of the space $\mathscr{F}$ of all immersed closed curves on $F$. However, as far as I know, this group is not calculated in the literature. In this paper we explicitly calculate it. The knowledge of its properties allowed me [9] to generalize in a natural way Arnold's invariants to the case of generic curves on an arbitrary surface. (The results of the well known paper by S. Smale [8], where he calculated homotopy groups of the space of all immersed closed curves with the fixed initial point and the velocity vector at it, are not sufficient for this purpose.)

When the work described in this paper was complete and submitted to Mathematica Scandinavica, I received a preprint of A. Inshakov [5] containing similar results obtained by him independently. (Later the preprint of Inshakov was broken into two parts [6] and [7].)

Acknowledgements. I am deeply grateful to Oleg Viro and Tobias Ekholm for many enlightening discussions. 


\section{Main results}

2.1. Basic definitions. A curve is a smooth immersion of an oriented circle $S^{1}$ into a (smooth) surface $F$. For a surface $F$ we denote by $\mathscr{F}$ the space of all curves on $F$.

Two curves $s_{0}$ and $s_{1}$ are said to be regularly homotopic, if there exists a homotopy $H: S^{1} \times I \rightarrow F$ such that $H(t \times 0)=s_{0}(t), H(t \times 1)=s_{1}(t)$, and $H(\bullet \times x)$ is an immersion for every $x \in I$. This means that $s_{0}$ and $s_{1}$ are in the same connected component of $\mathscr{F}$.

Two (oriented) curves with a tangency point, at which the velocity vectors of the two curves are pointing in the same direction, are said to be direct tangent to each other at this point.

For a surface $F$ we denote by ST $F$ the spherical tangent bundle of $F$ and by $\mathrm{pr}: \mathrm{ST} F \rightarrow F$ the corresponding locally trivial $S^{1}$-fibration.

For a curve $\xi$ on $F$ we denote by $\vec{\xi}$ its lifting to ST $F$, which maps every point $t \in S^{1}$ to the direction of the velocity vector of $\xi$ at $t$.

We fix a point $a$ on $S^{1}$. Then a curve $\xi$ represents an element of $\pi_{1}(F, \xi(a))$, and $\vec{\xi}$ represents an element of $\pi_{1}(\mathrm{ST} F, \vec{\xi}(a))$. When there is no ambiguity, we denote these two elements by $\xi$ and $\vec{\xi}$ respectively.

2.2. Fundamental group of the space of curves on an orientable surface. For orientable surfaces the group $\pi_{1}(\mathscr{F}, \xi)$ appears to be much simpler than for nonorientable surfaces.

THeOREM 2.2.1. Let $F=S^{2}$ and let $\xi$ be a curve on $S^{2}$. Then $\pi_{1}(\mathscr{F}, \xi)=Z_{2}$.

THEOREM 2.2.2. Let $F=T^{2}$ (torus) and let $\xi$ be a curve on $T^{2}$. Then $\pi_{1}(\mathscr{F}, \xi)=\mathbf{Z} \oplus \mathbf{Z} \oplus \mathbf{Z}$.

Theorem 2.2.3. Let $F \neq S^{2}, T^{2}$ be an orientable surface (not necessarily compact) and let $\xi$ be a curve on $F$.

I. If $\xi$ represents a homotopically nontrivial loop on $F$, then $\pi_{1}(\mathscr{F}, \xi)=\mathbf{Z} \oplus \mathbf{Z}$.

II. If $\xi$ represents a homotopically trivial loop on $F$, then $\pi_{1}(\mathscr{F}, \xi)=\pi_{1}(\mathrm{ST} F)$.

The proofs of Theorems 2.2.1., 2.2.2. and 2.2.3. are given in Subsections 3.2., 3.3., and 3.6., respectively.

2.3 Fundamental group of the space of curves on a nonorientable surface.

Theorem 2.3.1. Let $F=\mathrm{R} P^{2}$ and let $\xi$ be a curve on $\mathrm{R} P^{2}$. Then $\pi_{1}(\mathscr{F}, \xi)=Z_{4}$.

THeORem 2.3.2. Let $F=K$ (Klein bottle) and let $\xi$ be a curve on $K$.

I. If $\xi$ represents an orientation preserving loop on $K$, then $\pi_{1}(\mathscr{F}, \xi)=\pi_{1}(\mathrm{ST} K)$, provided that $\vec{\xi}=b^{2 l}$ in $\pi_{1}(\mathrm{ST} K, \vec{\xi}(a))$ for some 
$b \in \pi_{1}(\mathrm{ST} K, \vec{\xi}(a))$ projecting to an orientation reversing loop on $K$, and $\pi_{1}(\mathscr{F}, \xi)=\mathbf{Z} \oplus \mathbf{Z} \oplus \mathbf{Z}$ otherwise.

II. If $\xi$ represents an orientation reversing loop on $K$, then $\pi_{1}(\mathscr{F}, \xi)$ is isomorphic to $\mathbf{Z}$.

The following construction will be needed for a description of $\pi_{1}(\mathscr{F}, \xi)$ for $\xi$ representing a homotopically nontrivial loop on $F$ and $F \neq \mathrm{R} P^{2}, K$.

Let $F \neq \mathrm{R} P^{2}, K$ be a surface (not necessarily compact), and let $\xi$ be a curve on $F$ such that $\xi \neq 1 \in \pi_{1}(F, \xi(a))$. Let $f \in \pi_{1}(\operatorname{ST} F, \vec{\xi}(a))$ be the homotopy class of an oriented fiber of the $S^{1}$-fibration pr: STF $\rightarrow F$.

One can show, that there exists a unique maximal Abelian subgroup $G_{\xi}<\pi_{1}(F, \xi(a))$ containing $\xi \in \pi_{1}(F, \xi(a))$, and that this $G_{\xi}$ is isomorphic to $\mathrm{Z}$ (see also Proposition 3.1.8.). Let $g$ be its generator. Consider a curve $g_{\xi}$ direct tangent to $\xi$ at $\xi(a)$, which realizes $g \in \pi_{1}(F, \xi(a))$.

One can show, that $\vec{\xi} \in \pi_{1}(\mathrm{ST} F, \vec{\xi}(a))$ can be presented in the unique way as $\vec{g}_{\xi}^{k} f^{l} \in \pi_{1}(\mathrm{ST} F, \vec{\xi}(a))$ (see also the Proof of Theorem 2.3.3.).

THEOREM 2.3.3. Let $F \neq \mathrm{R} P^{2}, K$ be a nonorientable surface (not necessarily compact) and let $\xi$ be a curve on $F$.

I. If $\xi$ represents an orientation reversing loop on $F$, then $\pi_{1}(\mathscr{F}, \xi)=\mathbf{Z}$.

II. If $\xi$ represents a homotopically nontrivial orientation preserving loop on $F$ then:

a) $\pi_{1}(\mathscr{F}, \xi)=\mathbf{Z} \oplus \mathbf{Z}$, provided that $g_{\xi}$ is an orientation preserving loop on $F$, or that $g_{\xi}$ is an orientation reversing loop and $\vec{\xi}=\left(\vec{g}_{\xi}\right)^{2 k} f^{l}$ for some nonzero $k$ and $l$.

b) $\pi_{1}(\mathscr{F}, \xi)=\pi_{1}(K)$, provided that $g_{\xi}$ is an orientation reversing loop and $\vec{\xi}=\left(\vec{g}_{\xi}\right)^{2 k}$ for some nonzero $k$.

III. If $\xi$ represents a homotopically trivial loop, then:

a) $\pi_{1}(\mathscr{F}, \xi)$ is isomorphic to the subgroup of $\pi_{1}(\mathrm{ST} F)$ consisting of all the elements, which project to orientation preserving loops on $F$, provided that $\vec{\xi}$ is a homotopically nontrivial loop in STF. (This means, cf. 3.1.2, that $\xi$ is not regularly homotopic to the figure eight curve.)

b) $\pi_{1}(\mathscr{F}, \xi)=\pi_{1}(\mathrm{ST} F)$, provided that $\vec{\xi}$ is a homotopically trivial loop in $\mathrm{STF}$. (This means, cf. 3.1.2, that $\xi$ is regularly homotopic to the figure eight curve.)

The proofs of Theorems 2.3.1., 2.3.2. and 2.3.3. are given in Subsections 3.4., 3.5. and 3.6., respectively.

2.4. Higher homotopy groups of the space of curves.

THEOREM 2.4.1. Let $F$ be a surface (not necessarily compact or orientable) and let $\xi$ be a curve on $F$. 
I. If $F$ is equal to $S^{2}$ or $\mathrm{R} P^{2}$, then $\pi_{2}(\mathscr{F}, \xi)=\mathrm{Z}$ and $\pi_{n}(\mathscr{F}, \xi)=$ $\pi_{n}\left(S^{2}\right) \oplus \pi_{n+1}\left(S^{2}\right), n \geq 3$.

II. If $F \neq S^{2}, \mathrm{R} P^{2}$, then $\pi_{n}(\mathscr{F}, \xi)=0, n \geq 2$.

For the Proof of Theorem 2.4.1. see Subsection 3.7.

\section{Proofs}

\subsection{Some useful facts and technical Lemmas.}

Lemma 3.1.1. Let $F$ be a surface, let $\mathrm{ST} F$ be its spherical tangent bundle and let $p \in \mathrm{ST} F$ be a point. Let $f \in \pi_{1}(\mathrm{ST} F, p)$ be the class of an oriented (in some way) fiber of the $S^{1}$-fibration $\mathrm{pr}: \mathrm{ST} F \rightarrow F$.

If $\alpha \in \pi_{1}(\mathrm{ST} F, p)$ is a loop, which projects to an orientation preserving loop on $F$, then

$$
\alpha f=f \alpha .
$$

If $\alpha \in \pi_{1}(\mathrm{ST} F, p)$ is a loop, which projects to an orientation reversing loop on $F$, then

$$
\alpha f=f^{-1} \alpha .
$$

The proof of this Lemma is straightforward.

3.1.2. (Parametric h-principle.) The parametric $h$-principle, see [3] page 16, implies that $\mathscr{F}$ is weak homotopy equivalent to the space $\Omega \mathrm{ST} F$ of free loops in ST $F$. The corresponding mapping $h: \mathscr{F} \rightarrow \Omega \mathrm{ST} F$ sends an immersion $\xi \in \mathscr{F}$ to a loop $\vec{\xi} \in \Omega \mathrm{ST} F$ by mapping a point $y \in S^{1}$ to the point in $\mathrm{ST} F$ corresponding to the velocity vector of $\xi$ at $y$.

3.1.3. Relations between the homotopy groups of the space STF and of the space of free loops in ST $F$. Let $b$ be a point in ST $F$. We denote by $\Omega_{b} \mathrm{ST} F$ the space of all loops in $\mathrm{ST} F$ based at $b$.

Let $\Omega \mathrm{ST} F$ be the space of all free loops in ST $F$ and let $\lambda$ be a fixed element of $\Omega$ STF. Fix a point $a$ on $S^{1}$.

Let $t: \Omega \mathrm{ST} F \rightarrow \mathrm{ST} F$ be the mapping, which sends $\omega \in \Omega \mathrm{ST} F$ to $\omega(a) \in \mathrm{ST} F$. One verifies that $t$ is a Serre fibration with the fiber isomorphic to the space of loops based at the corresponding point.

This fibration gives rise to the following long exact sequence:

$$
\cdots \stackrel{\partial}{\rightarrow} \pi_{n}\left(\Omega_{\lambda(a)} \mathrm{ST} F, \lambda\right) \stackrel{i n_{*}}{\rightarrow} \pi_{n}(\Omega \mathrm{ST} F, \lambda) \stackrel{t_{*}}{\rightarrow} \pi_{n}(\mathrm{ST} F, \lambda(a)) \stackrel{\partial}{\rightarrow} \cdots
$$

The following statement is well known.

3.1.4. Let $\lambda$ be a loop in STF (not necessarily contractible) and let $a$ be a fixed point on $S^{1}$, then for any $n \geq 1: \pi_{n}\left(\Omega_{\lambda(a)} \operatorname{ST} F, \lambda\right)=\pi_{n+1}(\operatorname{ST} F, \lambda(a))$. 
Lemma 3.1.5. (Cf. V.L. Hansen [4]) The group $\pi_{1}(\Omega \mathrm{ST} F, \lambda)$ is isomorphic to $Z(\lambda)$, the centralizer of $\lambda \in \pi_{1}(\mathrm{ST} F, \lambda(a))$.

3.1.6. Proof of Lemma 3.1.5. Let $t: \Omega \mathrm{ST} F \rightarrow \mathrm{ST} F$ be the mapping described above. A Proposition proved by V.L. Hansen [4] says that: if $X$ is a topological space with $\pi_{2}(X)=0$, then $\pi_{1}(\Omega X, \omega)$ is isomorphic to $Z(\omega)<\pi_{1}(X, \omega(a))$. (Here $\Omega X$ is the space of free loops in $X$ and $\omega$ is an element of $\Omega X$.) One can verify that $\pi_{2}(\mathrm{ST} F)=0$ for any surface $F$. Thus, we get that $\pi_{1}(\Omega \mathrm{ST} F, \lambda)$ is isomorphic to $Z(\lambda)<\pi_{1}(\mathrm{ST} F, \lambda(a))$. From the proof of the Hansen's Proposition it follows that the isomorphism is induced by $t_{*}$.

The following statement is an immediate consequence of Lemma 3.1.5 and the $h$-principle.

Corollary 3.1.7. Let $F$ be a surface and let $\xi$ be a curve on $F$, then $\pi_{1}(\mathscr{F}, \xi)$ is isomorphic to $Z(\vec{\xi})$, the centralizer of $\vec{\xi} \in \pi_{1}(\operatorname{ST} F, \vec{\xi}(a))$.

Lemma 3.1.8. Let $F \neq S^{2}, T^{2}$ (torus), $\mathrm{R} P^{2}, K$ (Klein bottle) be a surface (not necessarily compact or orientable) and let $G^{\prime}$ be a nontrivial commutative subgroup of $\pi_{1}(F)$. Then $G^{\prime}$ is infinite cyclic and there exists a unique maximal infinite cyclic group $G<\pi_{1}(F)$ such that $G^{\prime}<G$.

3.1.9. Proof of Lemma 3.1.8. It is well known that any closed $F$, other than $S^{2}, T^{2}, \mathrm{R} P^{2}, K$, admits a hyperbolic metric of a constant negative curvature, which is induced from the universal covering of it by the hyperbolic plane $H$. The Theorem by A. Preissman (see [2] pp. 258-265) says that if $M$ is a compact Riemannian manifold with a negative curvature, then any nontrivial Abelian subgroup $G^{\prime}<\pi_{1}(M)$ is isomorphic to Z. Thus if $F \neq S^{2}, T^{2}, \mathrm{R} P^{2}, K$ is closed, then any nontrivial commutative $G^{\prime}<\pi_{1}(F)$ is infinite cyclic.

The proof of the Preissman's Theorem given in [2] is based on the fact, that if $\alpha, \beta \in \pi_{1}(M)$ are nontrivial commuting elements, then there exists a geodesic in $\bar{M}$ (the universal covering of $M$ ), which is mapped to itself under the action of these elements considered as deck transformations on $\bar{M}$. Moreover, these transformations restricted to the geodesic act as translations. This implies, that if $F \neq S^{2}, T^{2}, \mathrm{R} P^{2}, K$ is a closed surface, then there exists a unique maximal infinite cyclic $G<\pi_{1}(F)$ such that $G^{\prime}<G$. This gives the proof of the Lemma for closed $F$.

If $F$ is not closed, then this statement is also true, because in this case $F$ is homotopy equivalent to a bouquet of circles.

3.2. Proof of Theorem 2.2.1. From the exact homotopy sequence of the 
fibration pr: STS $S^{2} \rightarrow S^{2}$ we get that $\pi_{1}\left(\mathrm{STS}^{2}\right)=\mathrm{Z}_{2}$. Corollary 3.1.7. implies that $\pi_{1}(\mathscr{F}, \xi)=\mathbf{Z}_{2}$.

3.3. Proof of Theorem 2.2.2. From the exact homotopy sequence of the fibration $\mathrm{pr}: \mathrm{ST} T^{2} \rightarrow T^{2}$ and identity (1) we get that $\pi_{1}\left(\mathbf{S T} T^{2}\right)=\mathbf{Z} \oplus \mathbf{Z} \oplus \mathbf{Z}$. Corollary 3.1.7. implies that $\pi_{1}(\mathscr{F}, \xi)=\pi_{1}\left(\mathbf{S T} T^{2}\right)=$ $\mathbf{Z} \oplus \mathbf{Z} \oplus \mathbf{Z}$.

3.4. Proof of Theorem 2.3.1. From the exact homotopy sequence of the fibration pr: $\operatorname{STR} P^{2} \rightarrow \mathrm{R} P^{2}$ we get that $\pi_{1}\left(\mathrm{STR} P^{2}\right)=\mathrm{Z}_{4}$. Corollary 3.1.7. implies that $\pi_{1}(\mathscr{F}, \xi)=\mathbf{Z}_{4}$.

3.5. Proof of Theorem 2.3.2. Corollary 3.1.7. says that $\pi_{1}(\mathscr{F}, \xi)=$ $Z(\vec{\xi})<\pi_{1}(\mathrm{ST} K, \vec{\xi}(a))$.

Consider $K$ as a quotient of a rectangle modulo the identification on its sides shown in Figure 1. We can assume that $\xi(a)$ coincides with the image of a corner of the rectangle, and that $\xi$ and the side $c$ of the rectangle are direct tangent at this point. Let $g$ and $h$ be the curves such that: $\vec{\xi}(a)=\vec{g}(a)=\vec{h}(a)$, $g=c \in \pi_{1}(K, \xi(a))$ and $h=d \in \pi_{1}(K, \xi(a))$. (Here $c$ and $d$ are the elements of $\pi_{1}(K)$ realized by the images of the sides of the rectangle used to construct $K$, see Figure 1.)

One can show that:

$$
\pi_{1}(\mathrm{ST} K, \vec{\xi}(a))=\left\{\vec{g}, \vec{h}, f \mid \vec{h} \vec{g}^{ \pm 1}=\vec{g}^{\mp 1} \vec{h}, \vec{h} f^{ \pm 1}=f^{\mp 1} \vec{h}, \vec{g} f=f \vec{g}\right\} .
$$

The second and the third relations in this presentation follow from (1) and (2). To get the first relation one notes that the identity $d c^{ \pm 1}=c^{\mp 1} d \in \pi_{1}(K, \xi(a))$ implies that $\vec{h} \vec{g}^{ \pm 1}=\vec{g}^{\mp 1} \vec{h} f^{k}$ for some $k \in$ Z. But $\vec{h}^{2}$ commutes with $\vec{g}$, since they can be lifted to ST $T^{2}$ the fundamental group of which is Abelian. Hence $k=0$.

Relations present on the products of $f, \vec{g}, \vec{h}$ imply that any element of $\pi_{1}(\mathrm{ST} K, \vec{\xi}(a))$ can be presented as $\vec{g}^{k} \vec{h}^{l} f^{m}$ for some $k, l, m \in \mathbf{Z}$. Using this relations we calculate $Z(\vec{\xi})=\pi_{1}(\mathscr{F}, \xi)$.

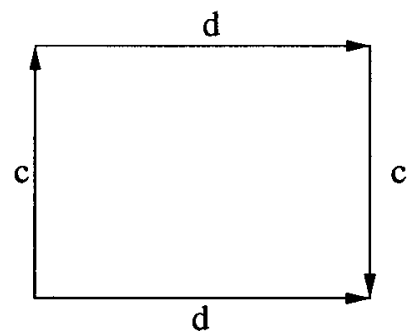

Figure 1 
This group appears to be:

a) The whole group $\pi_{1}(\mathrm{ST} K, \vec{\xi}(a))$, provided that $\vec{\xi}=\vec{h}^{2 l}$ for some $l \in \mathrm{Z}$.

b) An isomorphic to $\mathbf{Z} \oplus \mathbf{Z} \oplus \mathbf{Z}$ subgroup of $\pi_{1}(\mathrm{ST} K, \vec{\xi}(a))$, provided that $\vec{\xi}=\vec{g}^{k} \vec{h}^{2 l} f^{m}$ for some $k, l, m \in \mathbf{Z}$ such that $k \neq 0$ or $m \neq 0$. This subgroup is generated by $\left\{\vec{g}, \vec{h}^{2}, f\right\}$.

c) An isomorphic to Z subgroup of $\pi_{1}(\mathrm{ST} K, \vec{\xi}(a))$, provided that $\vec{\xi}=\vec{g}^{k} \vec{h}^{2 l+1} f^{m}$ for some $k, l, m \in \mathrm{Z}$. This subgroup is generated by $\alpha_{\xi}=\vec{g}^{k} \vec{h} f^{m}$. (Note that $\alpha_{\xi}^{2}=\vec{h}^{2}$, and $\vec{\xi}=\alpha_{\xi}^{2 l+1}$.)

Now the statement of the Theorem is a direct consequence of relations present.

3.6. Proof of Theorem 2.2.3. and Theorem 2.3.3. We are going to prove that the statement of Theorem 2.3.3. is true for any orientable surface $F \neq S^{2}, T^{2}$ and any nonorientable $F \neq \mathrm{R} P^{2}, K$. (We will see that $\vec{\xi} \in \pi_{1}(\operatorname{ST} F, \vec{\xi}(a))$ can be presented in the unique way as $\vec{g}_{\xi}^{k} f^{l} \in \pi_{1}(\operatorname{ST} F, \vec{\xi}(a))$ for any $F \neq S^{2}, \mathrm{R} P^{2}, T^{2}, K$.)

Clearly this gives a proof of Theorem 2.3.3. Theorem 2.2.3. is also an immediate consequence of this fact.

3.6.1. Proof of Theorem 2.2.3. and Theorem 2.3.3. in the case of $\xi \neq 1 \in \pi_{1}(F, \xi(a))$. Consider a subgroup $G^{\prime}$ of $\pi_{1}(F, \xi(a))$ generated by $\xi$. It is an infinite cyclic group (see 3.1.8). There is a unique (see 3.1.8.) maximal infinite cyclic group $G<\pi_{1}(F, \xi(a))$ such that $G^{\prime}<G$. Let $g$ be the generator of $G$. Let $g_{\xi}$ be a curve direct tangent to $\xi$ at $\xi(a)$ representing this $g$.

Corollary 3.1.7. says that $\pi_{1}(\mathscr{F}, \xi)$ is isomorphic to $Z(\vec{\xi})$. Take $\alpha \in Z(\vec{\xi})$. Since $\vec{\xi}$ and $\alpha$ commute in $\pi_{1}(\operatorname{ST} F, \vec{\xi}(a))$, we get that their images under the projection $\quad \mathrm{pr}_{*}: \pi_{1}(\mathrm{ST} F, \vec{\xi}(a)) \rightarrow \pi_{1}(F, \xi(a))$ commute in $\pi_{1}(F, \xi(a))$. Lemma 3.1.8 implies that these projections are in the subgroup $G$.

The kernel of the homomorphism $\mathrm{pr}_{*}$ is generated by $f$, the homotopy class of an oriented fiber of the $S^{1}$-fibration $\mathrm{pr}: \mathrm{ST} F \rightarrow F$. This fact and identities (1) and (2) show that there exist unique $k, l, m, n \in \mathbf{Z}$ such that $\vec{\xi}=\vec{g}_{\xi}^{k} f^{l}$ and $\alpha=\vec{g}_{\xi}^{m} f^{n}$.

Using identities (1) and (2) we can check for which values of $k, l, m, n$ the elements $\alpha$ and $\vec{\xi}$ commute. This allows us to calculate $Z(\vec{\xi})$. It turns out to be:

a) A group isomorphic to $\mathbf{Z} \oplus \mathbf{Z}$ generated by $\left\{\vec{g}_{\xi}, f\right\}$, provided that $g_{\xi}$ is an orientation preserving loop on $F$.

b) A group isomorphic to $Z$ generated by $\vec{g}_{\xi} f^{l}$, provided that $g_{\xi}$ is an orientation reversing loop, and $k$ is odd. (This means that $\xi$ represents an orientation reversing loop on $F$.) Note also, that in this case $\left(\vec{g}_{\xi} f^{l}\right)^{2}=\vec{g}_{\xi}^{2}$.

c) A group isomorphic to $\mathbf{Z} \oplus \mathbf{Z}$ generated by $\left\{\vec{g}_{\xi}^{2}, f\right\}$, provided that $g_{\xi}$ is an orientation reversing loop on $F, k \neq 0$ is even, and $l \neq 0$. 
d) A group isomorphic to $\pi_{1}(K)$ generated by $\left\{\vec{g}_{\xi}, f\right\}$, provided that $g_{\xi}$ is an orientation reversing loop on $F, k \neq 0$ is even, and $l=0$.

(Note that if $k=0$, then $\xi=1 \in \pi_{1}(\mathrm{ST} F, \xi(a))$, which contradicts to our assumption.)

This finishes the proof of the two theorems for this case.

3.6.2. Proof of Theorem 2.2.3. and Theorem 2.3.3. in the case of $\xi=1 \in \pi_{1}(F, \xi(a))$. From the exact homotopy sequence of the $S^{1}$-fibration pr : ST $F \rightarrow F$ we get that ker $\operatorname{pr}_{*}$ is generated by $f$, the homotopy class of the fiber. Since $\xi=1 \in \pi_{1}(F, \xi(a))$ we get that there exists a $k \in \mathbf{Z}$ such that $\vec{\xi}=f^{k}$. Lemma 3.7.1. says that $\pi_{1}(\mathscr{F}, \xi)$ is isomorphic to $Z(\vec{\xi})=$ $Z\left(f^{k}\right)<\pi_{1}(\mathrm{ST} F, \vec{\xi}(a))$.

For $k \neq 0$ identities (1) and (2) imply that $Z\left(f^{k}\right)$ coincides with the set of elements of $\pi_{1}(\mathrm{ST} F, \vec{\xi}(a))$, which project to orientation preserving loops on $F$. This finishes the proof of the Theorem for $\xi=1 \in \pi_{1}(F, \xi(a))$ and $\vec{\xi} \neq 1 \in \pi_{1}(\operatorname{ST} F, \vec{\xi}(a))$.

If $k=0$, then $\vec{\xi}=1 \in \pi_{1}(\operatorname{ST} F, \vec{\xi}(a))$. Thus $Z(\vec{\xi})=\pi_{1}(\operatorname{ST} F, \vec{\xi}(a))$. Hence, in this case $\pi_{1}(\mathscr{F}, \xi)=\pi_{1}(\operatorname{ST} F, \vec{\xi}(a))$.

3.7. Proof of Theorem 2.4.1. The proof of this Theorem is based on the following exact sequence, which was introduced in section 3.1.3.

$$
\cdots \stackrel{\partial}{\rightarrow} \pi_{n}\left(\Omega_{\lambda(a)} \mathrm{ST} F, \lambda\right) \stackrel{i n_{*}}{\rightarrow} \pi_{n}(\Omega \mathrm{ST} F, \lambda) \stackrel{t_{*}}{\rightarrow} \pi_{n}(\mathrm{ST} F, \lambda(a)) \stackrel{\partial}{\rightarrow} \cdots
$$

LemmA 3.7.1. If $F$ is equal to $S^{2}$ or $\mathrm{R} P^{2}$ and $n \geq 2$, then

$$
\pi_{n}(\Omega \mathrm{ST} F, \lambda)=\pi_{n}\left(\Omega_{\lambda(a)} \mathrm{ST} F, \lambda\right) \oplus \pi_{n}(\mathrm{ST} F, \lambda(a)) .
$$

3.7.2. Proof of Lemma 3.7.1. Fix $n>1$. We construct a homomorphism $g: \pi_{n}(\operatorname{ST} F, \lambda(a)) \rightarrow \pi_{n}(\Omega \mathrm{ST} F, \lambda)$ such that $t_{*} \circ g=\mathrm{id}_{\pi_{n}(\operatorname{ST} F, \lambda(a))}$. After this the exactness of the sequence (5) and the fact that higher homotopy groups are Abelian imply the statement of the Lemma.

We describe this construction for $F=\mathrm{R} P^{2}$. The construction of $g$ for $F=S^{2}$ can be easily deduced from this one.

From the exact homotopy sequence of the covering ST $S^{2} \rightarrow \operatorname{STR} P^{2}$ we get that $\pi_{n}\left(\operatorname{STR} P^{2}\right), n \geq 2$, is canonically isomorphic to $\pi_{n}\left(\operatorname{ST} S^{2}\right)$.

Take $s: S^{n} \rightarrow \mathrm{STR} P^{2}$, which represents a given element of $\pi_{n}\left(\operatorname{STR} P^{2}, \lambda(a)\right)$. Let $s^{\prime}: S^{n} \rightarrow \mathrm{ST}^{2}$ be the mapping which is a lifting of $s$ under the covering ST $S^{2} \rightarrow \mathrm{STR} P^{2}$. Fix an orientation of $S^{2}$. Then for every $x \in S^{n}$ the orientation of a small neighborhood of $\operatorname{pr} s^{\prime}(x) \in S^{2}$ induces an orientation of a small neighborhood of $\operatorname{pr} s(x) \in \mathrm{R} P^{2}$.

There is a unique isometric autodiffeomorphism $I_{x}$ of $\mathrm{R} P^{2}$ such that:

a) It maps $\operatorname{pr} s(*)$ to $\operatorname{pr} s(x)$. 
b) The differential of it sends $s(*)$ to $s(x)$.

c) The above described local orientation at $\operatorname{pr} s(x)$ coincides with the one induced by the differential of $I_{x}$ from the local orientation at $\operatorname{pr} s(*)$.

Let $\bar{s}: S^{n} \rightarrow \Omega \mathrm{STR} P^{2}$ be the mapping which sends $x \in S^{n}$ to $I_{x}(\lambda)$ (the translation of $\lambda$ by $I_{x}$ ).

Set the value of $g$ on the element of $\pi_{n}\left(\operatorname{STR} P^{2}, \lambda(a)\right)$ represented by $s$ to be the element of $\pi_{n}\left(\Omega \mathrm{STR} P^{2}, \lambda\right)$ represented by $\bar{s}$. A straightforward verification shows that this $g$ is the desired homomorphism from $\pi_{n}\left(\operatorname{STR} P^{2}, \lambda(a)\right)$ to $\pi_{n}\left(\Omega \operatorname{STR} P^{2}, \lambda\right)$.

This finishes the proof of Lemma 3.7.1.

3.7.3. One verifies that $\pi_{2}(\mathrm{ST} F)=0$ and $\pi_{n}(\mathrm{ST} F)=\pi_{n}\left(S^{2}\right), n \geq 3$, for $F$ equal to $S^{2}$ or $\mathrm{R} P^{2}$. Now Lemma 3.7.1, statement 3.1 .4 and the weak homotopy equivalence given by the $h$-principle (see 3.1.2) imply the first statement of the Theorem. (Note that $\pi_{3}\left(S^{2}\right)=$ Z.)

Statement 3.1.4. says that $\pi_{n}\left(\Omega_{\lambda(a)} \mathrm{ST} F, \lambda\right)=\pi_{n+1}(\mathrm{ST} F, \lambda(a))$. One verifies that $\pi_{n}(\mathrm{ST} F)=0, n \geq 2$ for $F \neq S^{2}, \mathrm{R} P^{2}$. The exactness of sequence (5) implies, that $\pi_{n}(\Omega \mathrm{ST} F, \lambda)=0, n \geq 2$. Using the weak homotopy equivalence given by the $h$-principle we get the second statement of the Theorem.

This finishes the proof of Theorem 2.4.1.

\section{REFERENCES}

1. V.I. Arnold, Plane curves, their invariants, perestroikas and classifications, Singularities and Bifurcations (V.I. Arnold, ed.) Adv. Sov. Math. 21 (1994), 39-91.

2. M. do Carmo, Riemannian Geometry, Birkhäuser, Boston (1992).

3. M. Gromov, Partial Differential Relations, Springer-Verlag Berlin Heidelberg (1986).

4. V.L. Hansen, On the fundamental group of the mapping space, Compositio Math. 28 (1974), 33-36.

5. A. V. Inshakov, Invarianty tipa $j^{+}, j^{-}$, st gladkih krivyh na dvumernyh mnogoobrazijah, preprint 1997.

6. A. V. Inshakov, Gomotopicheskie gruppy krivyh na dvumernyh mnogoobraziyah, Uspekhi Mat. Nauk 53 (2) (1998), 147-148, English translation: Homotopy groups of spaces of curves on two-dimensional manifolds, Russian Math. Surveys, 53 (1998), 390-391.

7. A. V. Inshakov, Invarianty tipa $j^{+}, j^{-}$, st gladkih krivyh na dvumernyh mnogoobrazijah, Functional Anal. Appl. 33 (2) (1999), 45-46, English translation: Invariants of the Types $j^{+}, j^{-}$and st for Smooth Curves on Two-dimensional Manifolds, Funct. Anal. Appl. 33 (1999), 189-198.

8. S. Smale, Regular curves on Riemannian manifolds, Trans. Amer. Math. Soc. 87 (1958), 492-513.

9. V. Tchernov, Arnold-type invariants of curves on surfaces and homotopy groups of the space of curves, Preprint, Dept. of Math., Uppsala Univ., Sweden, U.U.D.M. report 1997: 21.

10. V. Tchernov, Arnold-type invariants of curves on surfaces, J. Knot Theory Ramifications 8 (1999), 71-97.

DMATH G-66.4

EIDGENÖSISCHE TECHNISCHE HOCHSCHULLE

CH-8092 ZÜRICH

SWITZERLAND

e-mail address: chernov@math.ethz.ch 